\title{
Road Networks with Phase Transitions
}

\author{
Rinaldo M. Colombo* Paola Goatin ${ }^{\dagger}$ \\ Benedetto PiCCOLI ${ }^{\ddagger}$
}

January 22, 2009

\begin{abstract}
This paper is devoted to a hyperbolic 2-phase model for traffic flow on a network. The model is rigorously described and the existence of solutions is proved, without any restriction on the network geometry.
\end{abstract}

2000 Mathematics Subject Classification: 35L65, 90B20.

Key words and phrases: Hyperbolic Conservation Laws on Networks, Riemann Problem, Phase Transitions, Continuum Traffic Models

\section{Introduction}

The increasing interest of mathematicians in vehicular traffic flow modelling gave rise to various approaches, including the fluid-dynamic one. The latter considers traffic from a macroscopic point of view, focusing on the evolution of macroscopic variables, such as density and average velocity of cars. The first model of this type was introduced by Lighthill and Whitham, see [32], and independently by Richards, in [34], and is referred to as the LWR model. It is simply based on the conservation of the number of cars and on a speed-density relation, resulting in a single partial differential equation in conservation form.

Later on, several second order models, i.e. models with two equations, were considered as in the case of the well known Aw-Rascle model, see [1]. Other second order models can be found in [23, 25, 33, 35, 37, 38], while a third order model was presented in [26]. We refer the reader to $[5,20,27]$ for general presentations of the theory.

\footnotetext{
*Department of Mathematics, Via Branze 38, 25123 Brescia, Italy; E-mail: rinaldo@ing.unibs.it

${ }^{\dagger}$ Institut de Mathématiques de Toulon et du Var, I.S.I.T.V., Université du Sud - Toulon - Var, B.P. 56, 83162 La Valette du Var Cedex, France; E-mail: goatin@univ-tln.fr

${ }^{\ddagger}$ Istituto per le Applicazioni del Calcolo "M. Picone", Viale del Policlinico 137, 00161 Roma, Italy; E-mail: piccoli@iac.rm.cnr.it
} 
The present paper focuses on the model with phase transitions presented in [9] and extends it to road networks.

There are now many available results for the LWR model or the AwRascle model on networks, see [3, 7, 8, 17, 18, 19, 28, 29, 30]. However, this is the first result for a phase transition model on a network. The interest in such a theory is motivated also by other applications: data networks [16], supply chains [15, 24], air traffic management [4] and gas pipelines [2, 11, 12].

Our main result is the existence of weak solutions on the whole network for initial data in BV under a technical assumption. More precisely the latter asks for traffic to keep away from the zero velocity, see assumption $(\mathbf{H})$ in Section 6.1. Our construction is based on the wave-front tracking method, see $[6,10,14,20,31]$.

More precisely, first we consider Riemann problems at nodes, which are Cauchy problems with constant initial data on each road. Notice that the conservation of cars alone is not sufficient to single out a unique solution. Thus, one has to prescribe solutions for every initial data and we call the relative map a Riemann solver at nodes. Then, it is possible to construct approximate solutions using classical self-similar entropic solutions for Riemann problems inside roads and an assigned Riemann solver at junctions. To pass to the limit we rely on $\mathbf{B V}$ estimate on the density flux variation, and assumption $(\mathbf{H})$ is necessary to get $\mathbf{B V}$ bounds also on the density itself and the linearized momentum.

Following [21], we define the properties (Pr1), (Pr2) and (Pr3) of a Riemann solver (see Definition 6.3), which guarantee the needed bounds and thus the existence of solutions to Cauchy problems. These key properties are in particular satisfied by the Riemann solver R.S. $J$ introduced in Section 5.

The definition of R.S. $J$ for the case of the phase transition model is a nontrivial extension of the Riemann solver introduced in [8] for the LWR model. It prescribes a fixed distribution of traffic in outgoing roads, and then the maximization of the flux through the junction. In particular, the set of attainable states on a road, entering or exiting a junction, gives rise to non-convex sets of possible density fluxes. To have a continuous dependence of solutions we have to get convexity removing the metastable states from the attainable set. This choice is consistent with the idea that such states should appear in a transient situation, which should not happen at a junction.

The paper is organized as follows. In Section 2 we review the 2-phase model introduced in [9], recalling the classical Riemann solver. The Riemann problem at junctions is studied in sections 3, 4 and 5. Section 6 deals with the proof of existence of solutions on the whole network. Finally, an appendix is devoted to show that condition $(\mathbf{H})$ is indeed necessary for the boundedness of the total variation. 


\section{Traffic Flow Models with Phase-Transitions}

The following model was introduced in $[9, \S 2]$ :

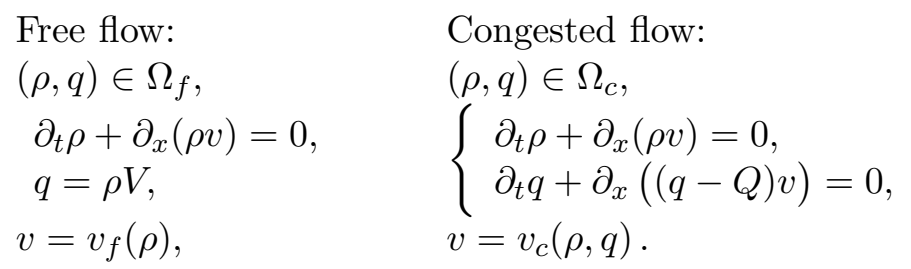

Here, $Q$ is a parameter of the road under consideration and the velocities $v_{f}, v_{c}$ are given by

$$
v_{f}(\rho)=V\left(1-\frac{\rho}{R}\right), \quad v_{c}(\rho, q)=\left(1-\frac{\rho}{R}\right) \frac{q}{\rho},
$$

where $V$ is the maximal mean velocity and $R$ is the maximal car density. The weighted linear momentum $q$ is originally motivated by gas dynamics. It approximates the real flux $\rho v$ for $\rho$ small compared to $R$.

We recall the basic features of model (2.1). In the free phase the characteristic speed is $\lambda(\rho)=V(1-2 \rho / R)$, while the information on the $2 \times 2$ system are collected in the following table:

$$
\begin{array}{ll}
r_{1}(\rho, q)=\left[\begin{array}{c}
\rho \\
q-Q
\end{array}\right], & r_{2}(\rho, q)=\left[\begin{array}{c}
R-\rho \\
\frac{R}{\rho} q
\end{array}\right], \\
\lambda_{1}(\rho, q)=\left(\frac{2}{R}-\frac{1}{\rho}\right) \cdot(Q-q)-\frac{Q}{R}, & \lambda_{2}(\rho, q)=v_{c}(\rho, q), \\
\nabla \lambda_{1} \cdot r_{1}(\rho, q)=2 \frac{Q-q}{R}, & \nabla \lambda_{2} \cdot r_{2}(\rho, q)=0, \\
\mathcal{L}_{1}\left(\rho ; \rho_{o}, q_{o}\right)=Q+\frac{q_{o}-Q}{\rho_{o}} \rho, & \mathcal{L}_{2}\left(\rho ; \rho_{o}, q_{o}\right)=\frac{\rho}{\rho_{o}} \frac{R-\rho_{o}}{R-\rho} q_{o}, \\
w_{1}(\rho, q)=v_{c}(\rho, q), & w_{2}(\rho, q)=\frac{q-Q}{\rho},
\end{array}
$$

where $r_{i}$ is the $i$-th right eigenvector, $\lambda_{i}$ the corresponding eigenvalue and $\mathcal{L}_{i}$ is the $i$-Lax curve. Shock and rarefaction curves coincide, hence system (2.1), right, belongs to Temple class [36]. The second characteristic field is linearly degenerate but the first has an inflection point along the curve $q=Q$.

It is reasonable to assume that if the initial data are entirely in the free (resp. congested) phase, then the solution remains in the free (resp. congested) phase for all time. Thus we are led to choose $\Omega_{f}$ (resp. $\Omega_{c}$ ) to be an invariant set for (2.1), left (resp. right). The resulting domain is given by

$\Omega_{f}=\left\{(\rho, q) \in[0, R] \times\left[0,+\infty\left[: v_{f}(\rho) \geq V_{f}, q=\rho \cdot V\right\}\right.\right.$,
$\Omega_{c}=\left\{(\rho, q) \in[0, R] \times\left[0,+\infty\left[: v_{c}(\rho, q) \leq V_{c}, \frac{q-Q}{\rho} \in\left[\frac{Q^{-}-Q}{R}, \frac{Q^{+}-Q}{R}\right]\right\}\right.\right.$, 
where $V_{f}$ and $V_{c}$ are the threshold speeds, i.e. above $V_{f}$ the flow is free and below $V_{c}$ the flow is congested. The parameters $\left.Q^{-} \in\right] 0, Q\left[\right.$ and $Q^{+} \in$ ]$Q,+\infty[$ depend on the environmental conditions and determine the width of the congested region.

For notational convenience, we introduce the following short form

$$
\partial_{t} \mathbf{u}+\partial_{x} \mathbf{f}(\mathbf{u})=0
$$

for the phase transition model (2.1), where

$$
\left\{\begin{array}{lll}
\mathbf{u}=(\rho, q) & \text { and } \quad \mathbf{f}(\mathbf{u})=\left(\rho v_{f}(\rho), q v_{f}(\rho)\right), & \text { if } \quad(\rho, q) \in \Omega_{f} \\
\mathbf{u}=(\rho, q) & \text { and } \quad \mathbf{f}(\mathbf{u})=\left(\rho v_{c}(\rho, q),(q-Q) v_{c}(\rho, q)\right), & \text { if } \quad(\rho, q) \in \Omega_{c}
\end{array}\right.
$$

\subsection{The Riemann Problem Outside Junctions}

We recall in this section the description of the classical Riemann solver for (2.1), i.e. the self-similar solution of the Cauchy problem

$$
\left\{\begin{array}{l}
\partial_{t} \mathbf{u}+\partial_{x} \mathbf{f}(\mathbf{u})=0 \\
\mathbf{u}_{0}(x)= \begin{cases}\mathbf{u}^{l} & \text { if } x<0, \\
\mathbf{u}^{r} & \text { if } x>0\end{cases}
\end{array}\right.
$$

If the initial data $\mathbf{u}^{l}, \mathbf{u}^{r}$ are in the same phase, the standard Lax solutions to the corresponding Riemann problem can be considered. Otherwise, following $[9, \S 3]$, admissible solutions are defined as follows.

Definition 2.1 If $\mathbf{u}^{l} \in \Omega_{f}$ and $\mathbf{u}^{r} \in \Omega_{c}$, then an admissible solution to (2.4) is a self-similar function $\mathbf{u}: \mathbb{R} \times\left[0,+\infty\left[\mapsto \Omega_{f} \cup \Omega_{c}\right.\right.$ such that, for some $\Lambda \in \mathbb{R}$, we have:

1. $\mathbf{u}(]-\infty, \Lambda t[) \subseteq \Omega_{f}$ and $\mathbf{u}(] \Lambda t,+\infty[) \subseteq \Omega_{c} ;$

2. the functions

$$
\begin{aligned}
& \mathbf{u}^{-}(x, t)= \begin{cases}\mathbf{u}(x, t) & \text { if } x<\Lambda t, \\
\mathbf{u}(\Lambda t-, t) & \text { if } x>\Lambda t,\end{cases} \\
& \mathbf{u}^{+}(x, t)= \begin{cases}\mathbf{u}(\Lambda t+, t) & \text { if } x<\Lambda t, \\
\mathbf{u}(x, t) & \text { if } x>\Lambda t,\end{cases}
\end{aligned}
$$

are Lax solutions to corresponding Riemann problems for (2.1) left, right, respectively;

3. the Rankine-Hugoniot condition

$$
\begin{aligned}
& \rho(\Lambda t+, t) v_{c}(\mathbf{u}(\Lambda t+, t))-\rho(\Lambda t-, t) v_{f}(\rho(\Lambda t+, t)) \\
= & \Lambda(\rho(\Lambda t+, t)-\rho(\Lambda t-, t))
\end{aligned}
$$

holds for all $t>0$. 
If $\mathbf{u}^{l} \in \Omega_{c}$ and $\mathbf{u}^{r} \in \Omega_{f}$, the conditions are obtained by exchanging the roles of $\Omega_{f}, \Omega_{c}$ and $v_{f}, v_{c}$.

Note that condition 3 above ensures that the total number of car is conserved across phase transitions.

Definition 2.1 does not assure uniqueness. We are then led to introduce the notion of consistency, see $[9, \mathbf{I}$ and $\mathbf{I I}]$.

Definition 2.2 Let $\mathcal{R}:\left(\mathbf{u}^{l}, \mathbf{u}^{r}\right) \mapsto \mathcal{R}\left(\mathbf{u}^{l}, \mathbf{u}^{r}\right)$ be a Riemann solver, i.e. $x \mapsto$ $\mathcal{R}\left(\mathbf{u}^{l}, \mathbf{u}^{r}\right)(x)$ is the solution of (2.4) computed at time $t=1$. $\mathcal{R}$ is consistent if the following two conditions hold for all $\mathbf{u}^{l}, \mathbf{u}^{m}, \mathbf{u}^{r} \in \Omega_{f} \cup \Omega_{c}$, and $\bar{x} \in \mathbb{R}:$

$$
\begin{aligned}
& \left.\begin{array}{l}
\mathcal{R}\left(\mathbf{u}^{l}, \mathbf{u}^{m}\right)(\bar{x})=\mathbf{u}^{m} \\
\mathcal{R}\left(\mathbf{u}^{m}, \mathbf{u}^{r}\right)(\bar{x})=\mathbf{u}^{m}
\end{array}\right\} \quad \Rightarrow \quad \mathcal{R}\left(\mathbf{u}^{l}, \mathbf{u}^{r}\right)=\left\{\begin{array}{l}
\mathcal{R}\left(\mathbf{u}^{l}, \mathbf{u}^{m}\right), \text { if } x<\bar{x}, \\
\mathcal{R}\left(\mathbf{u}^{m}, \mathbf{u}^{r}\right), \text { if } x \geq \bar{x},
\end{array}\right. \\
& \mathcal{R}\left(\mathbf{u}^{l}, \mathbf{u}^{r}\right)(\bar{x})=\mathbf{u}^{m} \Rightarrow\left\{\begin{array}{l}
\mathcal{R}\left(\mathbf{u}^{l}, \mathbf{u}^{m}\right)= \begin{cases}\mathcal{R}\left(\mathbf{u}^{l}, \mathbf{u}^{r}\right), & \text { if } x \leq \bar{x}, \\
\mathbf{u}^{m}, & \text { if } x>\bar{x},\end{cases} \\
\mathcal{R}\left(\mathbf{u}^{m}, \mathbf{u}^{r}\right)= \begin{cases}\mathbf{u}^{m}, & \text { if } x<\bar{x}, \\
\mathcal{R}\left(\mathbf{u}^{l}, \mathbf{u}^{r}\right), & \text { if } x \geq \bar{x} .\end{cases}
\end{array}\right.
\end{aligned}
$$

Essentially, (C1) states that whenever two solutions to two Riemann problems can be placed side by side, then their juxtaposition is again a solution to a Riemann problem. Condition (C2) is the viceversa.

We are now ready to construct the Riemann solver. Using Riemann coordinates $\left(w_{1}, w_{2}\right), \Omega_{c}=\left[0, V_{c}\right] \times\left[W_{2}^{-}, W_{2}^{+}\right]$. For $(\rho, q) \in \Omega_{f}$, we extend the corresponding Riemann coordinates $\left(w_{1}, w_{2}\right)$ as follows. Let $\tilde{\mathbf{u}}=(\tilde{\rho}, \tilde{\rho} V)$ be the point in $\Omega_{f}$ defined by $\tilde{\rho}=Q /\left(V-W_{2}^{-}\right)$. Define

$$
w_{1}=V_{f} \quad \text { and } \quad w_{2}= \begin{cases}V-Q / \rho & \text { if } \rho \geq \tilde{\rho} \\ v_{f}(\tilde{\rho})-v_{f}(\rho)+V-Q / \tilde{\rho} & \text { if } \rho<\tilde{\rho}\end{cases}
$$

so that, in the Riemann coordinates, $\Omega_{f}=\left\{V_{f}\right\} \times\left[W_{o}, W_{2}^{+}\right]$, see Figure 1, right. We consider several different cases:

(A) The data in (2.4) are in the same phase, i.e. they are either both in $\Omega_{f}$ or both in $\Omega_{c}$. Then the solution is the standard Lax solution to (2.1), left or to (2.1), right, and no phase boundary is present.

(B) $\mathbf{u}^{l} \in \Omega_{c}$ and $\mathbf{u}^{r} \in \Omega_{f}$. We consider the points $\mathbf{u}^{c} \in \Omega_{c}$ and $\mathbf{u}^{m} \in \Omega_{f}$ implicitly defined by

$$
\begin{aligned}
\left(1-\frac{\rho^{c}}{R}\right)\left(Q+w_{2}\left(\mathbf{u}^{l}\right) \rho^{c}\right) & =\rho^{c} V_{c}, \\
\left(1-\frac{\rho^{m}}{R}\right)\left(Q+w_{2}\left(\mathbf{u}^{l}\right) \rho^{m}\right) & =\rho^{m} V\left(1-\frac{\rho^{m}}{R}\right) .
\end{aligned}
$$



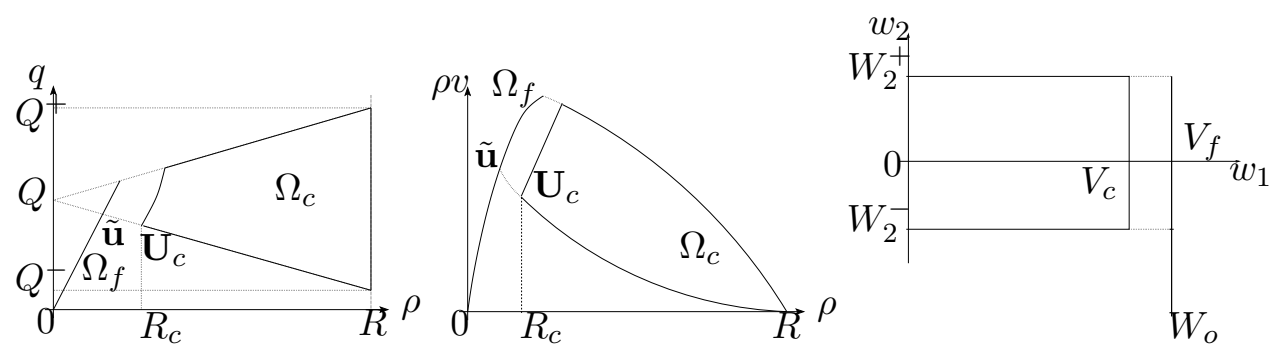

Figure 1: Coordinates used in the paper: left, the $(\rho, q)$ plane; middle, the fundamental diagram in the $(\rho, \rho v)$ plane; right, plane of the Riemann coordinate $\left(w_{1}, w_{2}\right)$.

If $w_{2}\left(\mathbf{u}^{l}\right)>0$, the solution is made of a 1-rarefaction from $\mathbf{u}^{l}$ to $\mathbf{u}^{c}$, a phase transition from $\mathbf{u}^{c}$ to $\mathbf{u}^{m}$ and a Lax wave from $\mathbf{u}^{m}$ to $\mathbf{u}^{r}$. If $w_{2}\left(\mathbf{u}^{l}\right) \leq 0$, we have a shock-like phase transition from $\mathbf{u}^{l}$ to $\mathbf{u}^{m}$ and a Lax wave from $\mathbf{u}^{m}$ to $\mathbf{u}^{r}$.

(C) $\mathbf{u}^{l} \in \Omega_{f}$ and $\mathbf{u}^{r} \in \Omega_{c}$ with $w_{2}\left(\mathbf{u}^{l}\right) \in\left[W_{2}^{-}, W_{2}^{+}\right]$. Consider the points $\mathbf{u}^{c}$ and $\mathbf{u}^{m} \in \Omega_{c}$ implicitly defined by

$$
\begin{aligned}
\left(1-\frac{\rho^{c}}{R}\right)\left(Q+w_{2}\left(\mathbf{u}^{l}\right) \rho^{c}\right) & =\rho^{c} V_{c}, \\
\left(1-\frac{\rho^{m}}{R}\right)\left(Q+w_{2}\left(\mathbf{u}^{l}\right) \rho^{m}\right) & =\rho^{m} w_{1}\left(\mathbf{u}^{r}\right) .
\end{aligned}
$$

If $w_{2}\left(\mathbf{u}^{l}\right)>0$, the solution is made of a shock-like phase transition from $\mathbf{u}^{l}$ to $\mathbf{u}^{m}$ and a 2-contact discontinuity $\mathbf{u}^{m}$ to $\mathbf{u}^{r}$. If $w_{2}\left(\mathbf{u}^{l}\right) \leq 0$, the solution displays a phase transition from $\mathbf{u}^{l}$ to $\mathbf{u}^{c}$, a 2-rarefaction from $\mathbf{u}^{c}$ to $\mathbf{u}^{m}$ and a 2-contact discontinuity $\mathbf{u}^{m}$ to $\mathbf{u}^{r}$.

(D) $\mathbf{u}^{l} \in \Omega_{f}$ with $w_{2}\left(\mathbf{u}^{l}\right)<W_{2}^{-}$and $\mathbf{u}^{r} \in \Omega_{c}$. Let $\mathbf{u}^{m} \in \Omega_{c}$ be the point on the lower boundary of $\Omega_{c}$ implicitly defined by

$$
\left(1-\frac{\rho^{m}}{R}\right)\left(Q+W_{2}^{-} \rho^{m}\right)=\rho^{m} w_{1}\left(\mathbf{u}^{r}\right),
$$

and consider the speed of the phase boundary joining $\mathbf{u}^{l} \in \Omega_{f}$ to $\mathbf{u}^{m} \in \Omega_{c}$

$$
\Lambda\left(\mathbf{u}^{l}, \mathbf{u}^{m}\right)=\frac{\rho^{l} v_{f}\left(\rho^{l}\right)-\rho^{m} w_{1}\left(\mathbf{u}^{r}\right)}{\rho^{l}-\rho^{m}} .
$$

Let $\mathbf{u}_{c}=\left(R_{c}, Q_{c}\right) \in \Omega_{c}$ be the point whose Riemann coordinates are $\left(V_{c}, W_{2}^{-}\right)$. If $\lambda_{1}\left(\mathbf{u}_{c}\right) \geq \Lambda\left(\mathbf{u}^{l}, \mathbf{u}_{c}\right)$, the solution is a phase transition from $\mathbf{u}^{l}$ to $\mathbf{u}_{c}$, a 1-rarefaction from $\mathbf{u}_{c}$ to $\mathbf{u}^{m}$ and a 2-contact discontinuity from $\mathbf{u}^{m}$ to $\mathbf{u}^{r}$. Otherwise: 
- If $\lambda_{1}\left(\mathbf{u}^{m}\right) \leq \Lambda\left(\mathbf{u}^{l}, \mathbf{u}^{m}\right)$, the solution is a phase transition from $\mathbf{u}^{l}$ to $\mathbf{u}^{m}$ followed by a 2-contact discontinuity from $\mathbf{u}^{m}$ to $\mathbf{u}^{r}$.

- If $\lambda_{1}\left(\mathbf{u}^{m}\right)>\Lambda\left(\mathbf{u}^{l}, \mathbf{u}^{m}\right)$, let $\mathbf{u}^{c}=\left(\rho^{c}, q^{c}\right) \in \Omega_{c}$ be defined by

$$
\lambda_{1}\left(\mathbf{u}^{c}\right)=\Lambda\left(\mathbf{u}^{l}, \mathbf{u}^{c}\right),
$$

i.e. $\rho^{c}$ is the bigger root of the equation

$$
\left(Q-Q^{-}\right) \rho^{2}-2 \rho^{l}\left(Q-Q^{-}\right) \rho+R^{2}\left(\rho^{l} v_{f}\left(\rho^{l}\right)-Q\right)+\rho^{l} R\left(2 Q-Q^{-}\right)=0
$$

and $q^{c}=Q-\rho^{c}\left(Q-Q^{-}\right) / R$. Then the solution shows a phase transition from $\mathbf{u}^{l}$ to $\mathbf{u}^{c}$, an attached 1-rarefaction from $\mathbf{u}^{c}$ to $\mathbf{u}^{m}$ and a 2-contact discontinuity from $\mathbf{u}^{m}$ to $\mathbf{u}^{r}$.

We now prove a simple result, very useful in the sequel for networks.

Proposition 2.3 Consider a phase transition $\left(\mathbf{u}_{l}, \mathbf{u}_{r}\right)$ with velocity $\lambda>0$ and $u_{r} \in \Omega_{c}$, then necessarily $\rho_{l} v_{f}\left(\rho_{l}\right)<\rho_{r} v_{c}\left(\rho_{r}\right)$. In other words the density flux on the right is bigger than the density flux on the left.

Consider now a phase transition $\left(\mathbf{u}_{l}, \mathbf{u}_{r}\right)$ with velocity $\lambda<0$ and $u_{r} \in \Omega_{c}$, then necessarily $\rho_{l} v_{f}\left(\rho_{l}\right)>\rho_{r} v_{c}\left(\rho_{r}\right)$. In other words the density flux on the left is bigger than the density flux on the right.

Proof. We prove the first statement, the proof of the second being similar. If $\rho_{l}<\tilde{\rho}$ then we have $\rho_{r} \geq R_{c}>\tilde{\rho}$, hence $\rho_{l}<\rho_{r}$. Since $\lambda$ is positive, the conclusion follows from the Rankine-Hugoniot condition (see 3. of Definition 2.1).

If $\rho_{l} \geq \tilde{\rho}$ then $\mathbf{u}_{r}$ belongs to the first family curve from $\mathbf{u}_{l}$ and $\rho_{l}<\rho_{r}$. Again the conclusion follows from the Rankine-Hugoniot condition.

\section{Road Network}

Following [20, Definition 4.1.1], by road network we mean a couple $(\mathcal{I}, \mathcal{J})$, where $\mathcal{I}$ is a finite collection of unidirectional roads and $\mathcal{J}$ is a set of junctions. Each road is modelled by real intervals $\left.I_{i}=\right] a_{i}, b_{i}[, i=1, \ldots, N$, while each $J$ consists of two sets $\operatorname{Inc}(J) \subset\{1, \ldots, N\}$ and $\operatorname{Out}(J) \subset\{1, \ldots, N\}$ corresponding to incoming and outgoing roads of $J$.

Fix a junction $J$ and assume for simplicity that $\operatorname{Inc}(J)=\{1, \ldots, n\}$ and $\operatorname{Out}(J)=\{n+1, \ldots, n+m\}$. A Riemann problem at $J$ is a Cauchy problem with initial data constant on each incoming and outgoing road:

$$
\left\{\begin{array}{l}
\partial_{t} \mathbf{u}_{i}+\partial_{x} \mathbf{f}\left(\mathbf{u}_{i}\right)=0, \quad i=1, \ldots, n+m \\
\mathbf{u}_{i}(0, x)=\mathbf{u}_{i, 0}
\end{array}\right.
$$


We now follow the same procedure used for classical Riemann problems on the real line: we first define admissible solutions at junction, state a consistency property and select a Riemann solver. All this, in view of the well posedness of the Cauchy problem.

Definition 3.1 If $\mathbf{u}_{i, o} \in \Omega_{f} \cap \Omega_{c}$ for $i=1, \ldots, n+m$, then an admissible solution to (3.6) is a self-similar function $\mathbf{u}: \mathbb{R} \times\left[0,+\infty\left[\mapsto\left(\Omega_{f} \cup \Omega_{c}\right)^{n+m}\right.\right.$ such that, for some $\hat{\mathbf{u}}_{1}, \ldots \hat{\mathbf{u}}_{n+m} \in \Omega_{f} \cap \Omega_{c}$, we have:

1. for every $i \in\{1, \ldots, n\},\left(\mathcal{R}\left(\mathbf{u}_{i, o}, \hat{\mathbf{u}}_{i}\right)\right)(x)=\hat{\mathbf{u}}_{i}$ for $x \geq 0$ and $\mathbf{u}_{i}(x)=$ $\left(\mathcal{R}\left(\mathbf{u}_{i, o}, \hat{\mathbf{u}}_{i}\right)\right)(x)$, for $x \leq 0 ;$

2. for every $i \in\{n+1, \ldots, n+m\},\left(\mathcal{R}\left(\hat{\mathbf{u}}_{i}, \mathbf{u}_{i, o}\right)\right)(x)=\hat{\mathbf{u}}_{i}$ for $x \leq 0$ and $\mathbf{u}_{i}(x)=\left(\mathcal{R}\left(\hat{\mathbf{u}}_{i}, \mathbf{u}_{i, o}\right)\right)(x)$, for $x \geq 0 ;$

3. $\sum_{i=1}^{n} \mathbf{f}_{1}\left(\hat{\mathbf{u}}_{i}\right)=\sum_{j=n+1}^{n+m} \mathbf{f}_{1}\left(\hat{\mathbf{u}}_{j}\right)$, where $\mathbf{f}_{1}$ is the first component of $\mathbf{f}$.

In other words, Definition 3.1 states that an admissible solution

1. consists of waves with negative speed in incoming roads;

2. consists of waves with positive speed in outgoing roads;

3. conserves the number of cars at $J$.

The above definition assigns a key role to the traces $\hat{\mathbf{u}}_{i}$ of admissible solutions at the junction. Once these values are known, the whole solution is uniquely determined thanks to 1 . and 2. in Definition 3.1. Therefore, following [20, Definition 4.2.2], in the case of the Riemann problem at a junction (3.6) we call Riemann Solver at $J$ the map

$$
\text { R.S.J: } \begin{array}{ccc}
\left(\Omega_{f} \cup \Omega_{c}\right)^{n+m} & \longrightarrow\left(\Omega_{f} \cup \Omega_{c}\right)^{n+m} \\
\left(\mathbf{u}_{1,0}, \ldots, \mathbf{u}_{n+m, 0}\right) & \longmapsto\left(\hat{\mathbf{u}}_{1}, \ldots, \hat{\mathbf{u}}_{n+m}\right)
\end{array}
$$

assigning to the initial data in (3.6) the trace $\hat{\mathbf{u}}_{1}, \ldots, \hat{\mathbf{u}}_{n+m}$ of the admissible solution at the junction.

In the case of (3.6), testing the consistency condition (C1) amounts to test its validity on $\mathcal{R}$. On the other hand, (C2) is substituted by the following condition, see [20, (ii), Definition 4.2.4].

Definition 3.2 We say that a Riemann solver at a Junction R.S.J is consistent at $J$ if

$$
\text { (CC) R.S.J }\left(R . S . J\left(\mathbf{u}_{1,0}, \ldots, \mathbf{u}_{n+m, 0}\right)\right)=R . S . J\left(\mathbf{u}_{1,0}, \ldots, \mathbf{u}_{n+m, 0}\right)
$$

for every $\left(\mathbf{u}_{1,0}, \ldots, \mathbf{u}_{n+m, 0}\right) \in\left(\Omega_{f} \cup \Omega_{c}\right)^{n+m}$. 


\section{The Riemann Problem at Junctions}

In what follows we assume that the parameters of model (2.1) satisfy

$$
\left(1-\frac{Q^{+}}{R V}\right) \cdot\left(\frac{Q^{+}}{Q}-1\right)<1
$$

The above condition guarantees that $\sup _{\mathbf{u} \in \Omega_{f} \cup \Omega_{c}} \lambda_{1}(\mathbf{u})<0$, hence 1-waves can only have negative speed, while 2 -waves always move with positive speed (see [13, formula (3.5)]). Note that, for instance, condition (4.7) is satisfied as soon as $Q^{+}$is sufficiently large, which can always be assumed without any loss of generality.

\subsection{Incoming Roads: Attainable Values at the Junction}

To satisfy condition 2. in Definition 3.1, only waves with negative speed can be produced on incoming roads. Thus, we determine all states which can be connected to an initial state (to the right) by waves with negative speed. In particular, we determine the maximum flux $\gamma_{i}^{\max }$ that can be reached from an initial datum $\mathbf{u}_{i, 0}=\left(\rho_{i, 0}, q_{i, 0}\right)$ by means of waves with negative speed only.

We start describing the sets of fluxes corresponding to states that can be connected to $\mathbf{u}_{i, 0}$ on the right using non positive waves only. We use the notations introduced in Section 2.1, Cases (B)-(D), where we set $\mathbf{u}_{i, 0}=\mathbf{u}^{l}$. Moreover, we introduce the velocities $V_{1}$ and $V_{2}$ defined as follows:

- $V_{1}:=v_{f}\left(\rho_{1}\right)$, where $\rho_{1} \in \Omega_{f}$ is the smaller root of the equation $\rho_{1} v_{f}\left(\rho_{1}\right)=R_{c} V_{c}$

- $V_{2}:=v_{f}\left(\rho_{2}\right)$, where $\rho_{2} \in \Omega_{f}$ is the smaller root of the equation

$$
\left(1-\frac{\rho_{2}}{R}\right)\left(Q+\frac{Q_{-}-Q}{R} \rho_{2}\right)=\rho_{2} V\left(1-\frac{\rho_{2}}{R}\right) .
$$

The notation below is explained in figures 2 and 3 . The sets of reachable fluxes are then given by

$$
\mathcal{O}_{i}= \begin{cases}{\left[0, \rho_{i, 0} v_{f}\left(\rho_{i, 0}\right)\right]} & \text { if } \mathbf{u}_{i, 0} \in \Omega_{f}, v_{f}\left(\rho_{i, 0}\right) \geq V_{1}, \\ {\left[0, R_{c} V_{c}\right] \cup\left\{\rho_{i, 0} v_{f}\left(\rho_{i, 0}\right)\right\}} & \text { if } \mathbf{u}_{i, 0} \in \Omega_{f}, V_{2} \leq v_{f}\left(\rho_{i, 0}\right) \leq V_{1} \\ {\left[0, \rho^{c} V_{c}\right] \cup\left\{\rho_{i, 0} v_{f}\left(\rho_{i, 0}\right)\right\}} & \text { if } \mathbf{u}_{i, 0} \in \Omega_{f}, v_{f}\left(\rho_{i, 0}\right) \leq V_{2} \\ {\left[0, \rho^{c} V_{c}\right] \cup\left\{\rho^{m} v_{f}\left(\rho^{m}\right)\right\}} & \text { if } \mathbf{u}_{i, 0} \in \Omega_{c}\end{cases}
$$

where we referred to the cases in Section 2.1 and for $i=1, \ldots, n$. Since the sets $\mathcal{O}_{i}$ are non convex, we do not take in account the metastable states in 

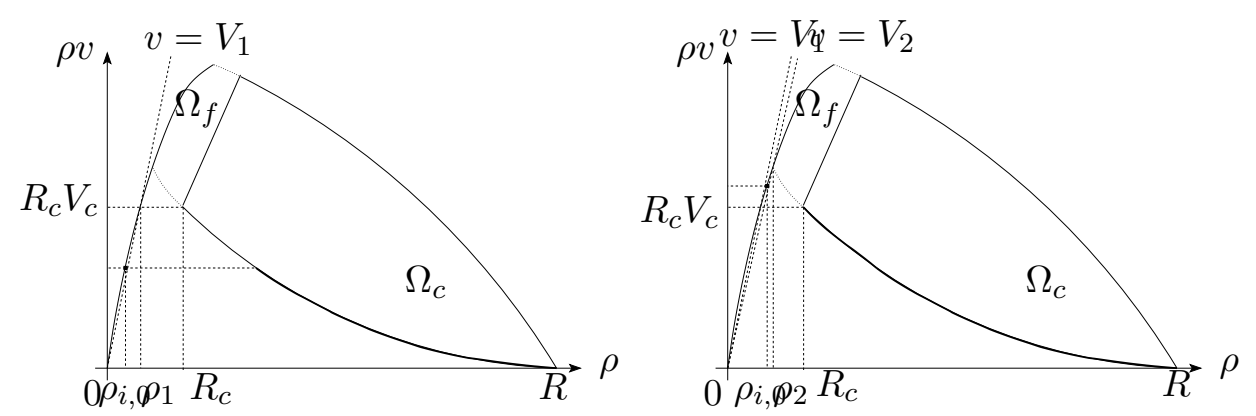

Figure 2: Notations used in the definition of $\mathcal{O}_{i}, i=1, \ldots, n$, first two cases.

the definition of the corresponding maximum fluxes:

$$
\gamma_{i}^{\max }=\left\{\begin{array}{lll}
\rho_{i, 0} v_{f}\left(\rho_{i, 0}\right) & \text { if } \mathbf{u}_{i, 0} \in \Omega_{f}, v_{f}\left(\rho_{i, 0}\right) \geq V_{1} \\
R_{c} V_{c} & \text { if } \mathbf{u}_{i, 0} \in \Omega_{f}, V_{2} \leq v_{f}\left(\rho_{i, 0}\right) \leq V_{1} & \text { (D) } \\
\rho^{c} V_{c} & \text { if } \mathbf{u}_{i, 0} \in \Omega_{f}, v_{f}\left(\rho_{i, 0}\right) \leq V_{2} & \text { (C) } \\
\rho^{c} V_{c} & \text { if } \mathbf{u}_{i, 0} \in \Omega_{c} & \text { (B) }
\end{array}\right.
$$

where we referred to the cases in Section 2.1.
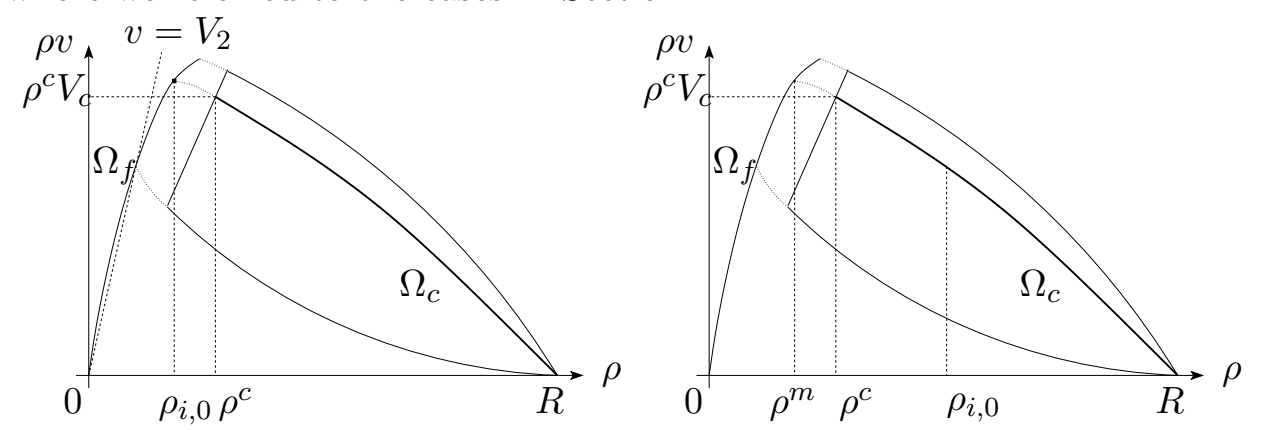

Figure 3: Notations used in the definition of $\mathcal{O}_{i}, i=1, \ldots, n$, cases $\mathbf{C}$ ) and (D).

Proposition 4.1 Given an initial datum $\mathbf{u}_{i, 0}$ on an incoming road and $\hat{\gamma} \epsilon$ $\left[0, \gamma_{i}^{\max }\right]$, there exists a unique $\hat{\mathbf{u}}_{i} \in \mathcal{O}_{i}$ such that the Riemann problem $\left(\mathbf{u}_{i, 0}, \hat{\mathbf{u}}_{i}\right)$ is solved by waves with negative speed and $\mathbf{f}_{1}\left(\hat{\mathbf{u}}_{i}\right)=\hat{\gamma}$.

\subsection{Outgoing Roads: Maximal Flux at the Junction}

To satisfy condition 3. in Definition 3.1, only waves with positive speed can be produced on outgoing roads. Thus we determine all states, and the corresponding set of fluxes, which can be connected to an initial state $\mathbf{u}_{j, 0}$ (to the left) using waves with positive speed.

We introduce the fluxes $F$ and $f^{\max }$ defined as follows (see Figure 4): 

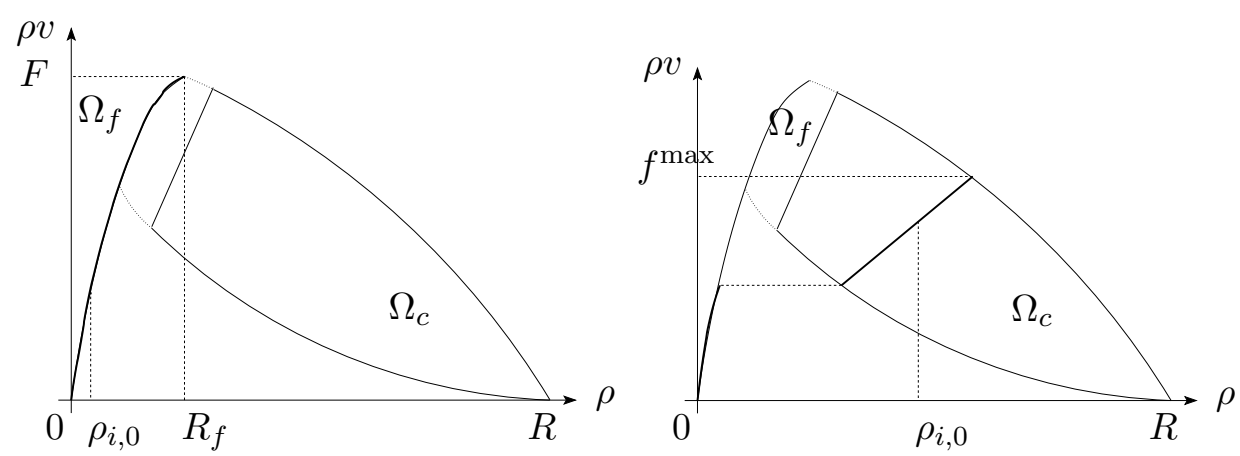

Figure 4: Notations used in the definition of $\mathcal{O}_{j}, j=n+1, \ldots, n+m$.

- $F=R_{f} v_{f}\left(R_{f}\right)=\max _{\rho \in \Omega_{f}} \rho v_{f}(\rho)>\max _{(\rho, q) \in \Omega_{c}} \rho v_{c}(\rho, q)$ is the maximal flux supported by the road;

- for $\mathbf{u}_{j, 0} \in \Omega_{c}, f^{\max }=f^{\max }\left(\mathbf{u}_{j, 0}\right)=\rho^{\max } v_{c}\left(\rho^{\max }, q^{\max }\right)$, where $\rho^{\max }$ is the bigger root of the equation

$$
\left(1-\frac{\rho^{\max }}{R}\right)\left(Q+\frac{Q_{+}-Q}{R} \rho^{\max }\right)=\rho^{\max } v_{c}\left(\rho_{j, 0}, q_{j, 0}\right),
$$

and $q^{\max }=Q+\rho^{\max }\left(Q_{+}-Q\right) / R$.

The sets of reachable fluxes are given by (see Figure 4)

$$
\mathcal{O}_{j}= \begin{cases}{[0, F]} & \text { if } \mathbf{u}_{j, 0} \in \Omega_{f}, \\ {\left[0, f^{\max }\right]} & \text { if } \mathbf{u}_{j, 0} \in \Omega_{c},\end{cases}
$$

for $j=n+1, \ldots, n+m$. Since the sets $\mathcal{O}_{j}$ are convex, the corresponding maximum fluxes are defined accordingly:

$$
\gamma_{j}^{\max }= \begin{cases}F & \text { if } \mathbf{u}_{j, 0} \in \Omega_{f}, \\ f^{\max } & \text { if } \mathbf{u}_{j, 0} \in \Omega_{c} .\end{cases}
$$

Proposition 4.2 Given an initial datum $\mathbf{u}_{j, 0}$ on an outgoing road and $\hat{\gamma} \in$ $\left[0, \gamma_{j}^{\max }\right]$, there exists a unique $\hat{\mathbf{u}}_{j} \in \mathcal{O}_{j}$ such that the Riemann problem $\left(\hat{\mathbf{u}}_{j}, \mathbf{u}_{j, 0}\right)$ is solved by waves with positive speed and $\mathbf{f}_{1}\left(\hat{\mathbf{u}}_{j}\right)=\hat{\gamma}$.

\section{The Riemann Solver at Junctions}

We define a Riemann solver similar to that introduced in [8] for vehicular traffic. 
First we need to define a suitable set of matrices. Consider the set

$$
\mathcal{A}:=\left\{A=\left\{a_{j i}\right\}_{i=1, \ldots, n, j=n+1, \ldots, n+m}: \begin{array}{c}
0<a_{j i}<1 \forall i, j, \\
\sum_{j=n+1}^{n+m} a_{j i}=1 \forall i
\end{array}\right\} .
$$

Let $\left\{e_{1}, \ldots, e_{n}\right\}$ be the canonical basis of $\mathbb{R}^{n}$. For every $i=1, \ldots, n$, we denote $H_{i}=\left\{e_{i}\right\}^{\perp}$. If $A \in \mathcal{A}$, then we write, for every $j=n+1, \ldots, n+m$, $a_{j}=\left(a_{j 1}, \ldots, a_{j n}\right) \in \mathbb{R}^{n}$ and $H_{j}=\left\{a_{j}\right\}^{\perp}$. Let $\mathcal{K}$ be the set of indexes $\mathbf{k}=\left(k_{1}, \ldots, k_{\ell}\right), 1 \leq \ell \leq n-1$, such that $0 \leq k_{1}<k_{2}<\cdots<k_{\ell} \leq n+m$ and for every $\mathbf{k} \in \mathcal{K}$ define

$$
H_{\mathbf{k}}:=\bigcap_{h=1}^{\ell} H_{k_{h}}
$$

Writing $\mathbf{1}=(1, \ldots, 1) \in \mathbb{R}^{n}$ and following [8] we define the set

$$
\mathfrak{N}:=\left\{A \in \mathcal{A}: \mathbf{1} \notin H_{\mathbf{k}}^{\perp} \text { for every } \mathbf{k} \in \mathcal{K}\right\} .
$$

Notice that, if $n>m$, then $\mathfrak{N}=\emptyset$. The matrices of $\mathfrak{N}$ give rise to a unique solution to Riemann problems at $J$.

1. Fix a matrix $A \in \mathfrak{N}$ and consider the closed, convex and not empty set

$$
\Lambda=\left\{\left(\gamma_{1}, \ldots, \gamma_{n}\right) \in \prod_{i=1}^{n}\left[0, \gamma_{i}^{\max }\right]: A \cdot\left(\gamma_{1}, \ldots, \gamma_{n}\right)^{T} \in \prod_{j=n+1}^{n+m}\left[0, \gamma_{j}^{\max }\right]\right\}
$$

2. Find the point $\left(\bar{\gamma}_{1}, \ldots, \bar{\gamma}_{n}\right) \in \Lambda$ which maximizes the function

$$
E\left(\gamma_{1}, \ldots, \gamma_{n}\right)=\gamma_{1}+\cdots+\gamma_{n}
$$

and define $\left(\bar{\gamma}_{n+1}, \ldots, \bar{\gamma}_{n+m}\right)^{T}:=A \cdot\left(\bar{\gamma}_{1}, \ldots, \bar{\gamma}_{n}\right)^{T}$. Since $A \in \mathfrak{N}$, the point $\left(\bar{\gamma}_{1}, \ldots, \bar{\gamma}_{n}\right)$ is uniquely defined.

3. For every $i \in\{1, \ldots, n\}$, set $\hat{\mathbf{u}}_{i}$ either by $\mathbf{u}_{i, 0}$ if $\mathbf{f}_{1}\left(\mathbf{u}_{i, 0}\right)=\bar{\gamma}_{i}$, or by the solution to $\mathbf{f}_{1}(\mathbf{u})=\bar{\gamma}_{i}$ given by Proposition 4.1. For every $j \in\{n+1, \ldots, n+m\}$, set $\hat{\mathbf{u}}_{j}$ either by $\mathbf{u}_{j, 0}$ if $\mathbf{f}_{1}\left(\mathbf{u}_{j, 0}\right)=\bar{\gamma}_{j}$, or by the solution to $\mathbf{f}_{1}(\mathbf{u})=\bar{\gamma}_{j}$ given by Proposition 4.2. Finally, set

$$
\text { R.S. } J\left(\mathbf{u}_{1,0}, \ldots, \mathbf{u}_{n+m, 0}\right)=\left(\hat{\mathbf{u}}_{1}, \ldots, \hat{\mathbf{u}}_{n+m}\right) .
$$

It is easy to verify that R.S. $J$ satisfies the consistency condition (CC). The following property of R.S. $J$ is used in the proof of Theorem 6.4 to bound the number of waves in the approximate solution. 
Lemma 5.1 Fix an equilibrium $\mathbf{u}_{0}=\left(\mathbf{u}_{1,0}, \ldots, \mathbf{u}_{n+m, 0}\right)$ for R.S.J corresponding to a matrix $A \in \mathfrak{N}$. Consider, for some $l \in\{1, \ldots, n+m\}$, $\mathbf{u}_{l} \in \Omega_{f} \cup \Omega_{c}$ such that the wave $\left(\mathbf{u}_{l}, \mathbf{u}_{l, 0}\right)$ has positive speed if $l \leq n$, or the wave $\left(\mathbf{u}_{l, 0}, \mathbf{u}_{l}\right)$ has negative speed if $l>n$ (the wave is approaching the junction). There exists a constant $\tilde{C}=\tilde{C}(A)>0$ such that for every $i \neq l$

$$
\left|\Gamma^{+}-\Gamma^{-}\right| \geq \tilde{C}\left|\mathbf{f}_{1}\left(\hat{\mathbf{u}}_{i}\right)-\mathbf{f}_{1}\left(\mathbf{u}_{i, 0}\right)\right|,
$$

where $\Gamma^{-}=\sum_{i=1}^{n} \mathbf{f}_{1}\left(\mathbf{u}_{i, 0}\right)$ and $\Gamma^{+}=\sum_{i=1}^{n} \mathbf{f}_{1}\left(\hat{\mathbf{u}}_{i}\right)$ are the through fluxes before and after the interaction, and:

$$
\left(\hat{\mathbf{u}}_{1}, \ldots, \hat{\mathbf{u}}_{n+m}\right)=R . S \cdot J\left(\mathbf{u}_{1,0}, \ldots, \mathbf{u}_{l}, \ldots, \mathbf{u}_{n+m, 0}\right) .
$$

Proof. If a wave is produced on road $i \neq l$ then the maximum point of $E$ computed at point 2. must change due to the interaction of the approaching wave. Since the directions of the faces of the set $\Lambda$, defined in (5.12), depend only on the matrix $A \in \mathfrak{N}$ and the solution for the flux lies on the boundary of $\Lambda$, we have that $\left|\Gamma^{+}-\Gamma^{-}\right|$is proportional to $\left|\mathbf{f}_{1}\left(\hat{\mathbf{u}}_{i}\right)-\mathbf{f}_{1}\left(\mathbf{u}_{i, 0}\right)\right|$.

\section{Existence of Solutions on the Whole Network}

To prove existence of solutions on the whole network, we first construct a sequence of approximate solutions via wave-front tracking and then pass to the limit using a BV bound on the density flux.

\subsection{Wave-Front Tracking and Statement of Main Result}

Since solutions to Riemann problems are given, we are able to construct piecewise constant approximations via wave-front tracking algorithm; see [6] for the general theory and $[20, \S 4.3]$ in the case of networks.

Roughly speaking a wave-front tracking solution is constructed as follows. Fix an initial datum $\mathbf{u}_{0}=\left(\mathbf{u}_{1,0}, \ldots, \mathbf{u}_{N, 0}\right)$ with bounded total variation on the whole network. For every $\nu \in \mathbb{N}$, one first discretizes the initial datum using a piecewise constant approximation $\mathbf{u}_{\nu, 0}$ with total variation bounded by the total variation of $\mathbf{u}_{0}$. Then Riemann problems on each road and inside the network are solved, replacing rarefaction waves by a collection of small rarefaction shocks of size at most $1 / \nu$. A solution is obtained for small times piecing together the solution to Riemann problems and is a weak solution up to the interaction of two waves or of a wave with a junction. Then a new Riemann problem is solved and so on.

To construct approximate solutions, one needs to bound the number of waves and of interactions. This is easily true for the scalar case on a real line, 
while for systems one has to rely on simplified Riemann solvers and nonphysical waves (see [6]), and for networks either on transfinite induction (see [20]), or on accurate estimates of waves number based also on variation estimates (see [21]). In this case we apply the latter method and obtain an approximate solution $\mathbf{u}_{\nu}$.

To pass to the limit in $\nu$ and construct a solution, one usually relies on bounds on the total variation of the solution or of the flux of the solution. In our case we obtain estimates on $\mathbf{f}_{1}\left(\mathbf{u}_{\nu}\right)$. To provide the needed estimates on $\rho_{\nu}$ and $q_{\nu}$ we make the following assumption:

(H) There exists a positive $\bar{v}$ such that the approximate solutions $\mathbf{u}_{\nu}=$ $\left(\mathbf{u}_{1, \nu}, \ldots, \mathbf{u}_{N, \nu}\right)$ attain values in $\widetilde{\Omega}=\Omega_{f} \cup\left\{(\rho, q) \in \Omega_{c}: v_{c}(\rho, q) \geq \bar{v}\right\}$.

Assumption $(\mathbf{H})$ may be verified by assuming conditions on the initial data and the traffic distribution coefficients. It is also verified as long as the traffic keeps away from the complete congestion, which is the standard situation in, say, highway traffic. For a discussion of continuum models in urban traffic, see [22].

Besides, another possible way to deal with $(\mathbf{H})$ is to modify the system so that the point of maximal density corresponds to a non zero traffic speed.

It is easy to verify the following:

Proposition 6.1 If assumption $(\boldsymbol{H})$ holds, there exists $C=C(\bar{v})$ such that, for every $\mathbf{u}_{1}, \mathbf{u}_{2} \in \widetilde{\Omega}$ belonging to the same phase, one has:

$$
\left|\rho_{1}-\rho_{2}\right| \leq C\left|\mathbf{f}_{1}\left(\mathbf{u}_{1}\right)-\mathbf{f}_{1}\left(\mathbf{u}_{2}\right)\right|, \quad\left|q_{1}-q_{2}\right| \leq C\left|\mathbf{f}_{1}\left(\mathbf{u}_{1}\right)-\mathbf{f}_{1}\left(\mathbf{u}_{2}\right)\right| .
$$

Therefore, under assumption $(\mathbf{H})$, a $\mathbf{B V}$ estimate on $\mathbf{f}_{1}\left(\mathbf{u}_{l, \nu}\right), l=1, \ldots, N$, ensures the estimates on the conserved variables, provided we give bounds on the number of phase boundaries in $\mathbf{u}_{l, \nu}$.

Our strategy to get a $\mathbf{B V}$ estimate on $\mathbf{f}_{1}\left(\mathbf{u}_{l, \nu}\right)$ is the following. We determine two basic properties (Pr2) and (Pr3) of the map R.S. $J$ which guarantee the desired estimates. These properties can be verified as in [21].

Consider a wave front tracking approximate solution $\mathbf{u}_{\nu}$ and define the functionals

$$
\begin{aligned}
\Gamma_{J}(t) & :=\sum_{i \in \operatorname{Inc}(J)} \mathbf{f}_{1}\left(\mathbf{u}_{i, \nu}\left(b_{i}-, t\right)\right) \\
T V_{f}(t) & :=\sum_{l=1}^{N} \operatorname{T.V} .\left(\mathbf{f}_{1}\left(\mathbf{u}_{l, \nu}(\cdot, t)\right)\right),
\end{aligned}
$$

where $J$ is a given junction and T.V. denotes the total variation with respect to the space variable. These functionals are well defined for every positive time and can vary only when a wave reaches a junction or when two waves interact in a road. Thus, we easily derive for every $t \geq 0$ the bound

$$
0 \leq \Gamma_{J}(t) \leq \# \operatorname{Inc}(J) F,
$$


where \#Inc(J) is the cardinality of $\operatorname{Inc}(J)$.

Definition 6.2 Let $\left(\mathbf{u}_{l}, \mathbf{u}_{r}\right)$ a wave interacting with $J$ from $I_{i}, i \in \operatorname{Inc}(J)$, then we say that the wave has decreasing flux if $\mathbf{f}_{1}\left(\mathbf{u}_{l}\right)<\mathbf{f}_{1}\left(\mathbf{u}_{r}\right)$ (i.e. the flux at the junction $J$ from road $I_{i}$ decreased because of the interaction).

Let $\left(\mathbf{u}_{l}, \mathbf{u}_{r}\right)$ a wave interacting with $J$ from $I_{j}, j \in O u t(J)$, then we say that the wave has decreasing flux if $\mathbf{f}_{1}\left(\mathbf{u}_{l}\right)>\mathbf{f}_{1}\left(\mathbf{u}_{r}\right)$ (i.e. the flux at the junction $J$ from road $I_{j}$ decreased because of the interaction).

Now we can state the two key properties of a Riemann solver, which ensures the necessary bounds on approximate solutions.

Definition 6.3 We say that a Riemann solver R.S.J at a junction $J$ has property $(\boldsymbol{P r} 1)$ if the solution depends only on the values $\gamma_{i}^{\max }, i \in \operatorname{Inc}(J)$, see (4.8), and $\gamma_{j}^{\max }, i \in \operatorname{Inc}(J)$, see (4.9).

We say that a Riemann solver R.S.J has property (Pr2) if there exists $C>0$ such that the following holds true. Assume $\mathbf{u}_{0}$ is an equilibrium at $J$, i.e. R.S.J $\left(\mathbf{u}_{0}\right)=\mathbf{u}_{0}$, a wave is interacting with $J$ and there is no other wave in the network. Denote with $T V_{f}^{-}$, resp. $T V_{f}^{+}$, the value of $T V_{f}$ before, resp. after, the interaction, similarly for $\Gamma_{J}$ and set $\Delta \Gamma_{J}=\left|\Gamma_{J}^{+}-\Gamma_{J}^{-}\right|$, then

$$
T V_{f}^{+}-T V_{f}^{-} \leq C \min \left\{T V_{f}^{-}, \Delta \Gamma_{J}\right\} .
$$

We say that R.S.J has property (Pr3) if the following holds true. Assume $\mathbf{u}_{0}$ is an equilibrium, i.e. R.S. ${ }_{J}\left(\mathbf{u}_{0}\right)=\mathbf{u}_{0}$, and a wave with decreasing flux is interacting with $J$. Denote with $\Gamma_{J}^{-}$, resp. $\Gamma_{J}^{+}$, the value of $\Gamma_{J}$ before, respectively after, the interaction. Then,

$$
\Gamma_{J}^{+} \leq \Gamma_{J}^{-}
$$

We can now state our main result.

Theorem 6.4 Consider a network $(\mathcal{I}, \mathcal{J})$, a Riemann solver R.S.J for every $J \in \mathcal{J}$ satisfying properties (Pr1), (Pr2) and (Pr3), an initial datum $\mathbf{u}_{0}$ on the network, with bounded total variation, and let $\mathbf{u}_{\nu}$ be a sequence of wave-front tracking approximate solutions. If $(\boldsymbol{H})$ holds true, then there exists the limit $\mathbf{u}$ of $\mathbf{u}_{\nu}$ in $L_{l o c}^{1}$ and $\mathbf{u}$ is a weak entropic solution on each road of the network with $\mathbf{u}_{0}$ as initial datum. Moreover, for every $J \in \mathcal{J}$ and for a.e. $t>0$ :

$$
R . S .{ }_{J}\left(\mathbf{u}_{J}(t)\right)=\mathbf{u}_{J}(t),
$$

where $\mathbf{u}_{J}=\left(\mathbf{u}_{i}\left(b_{i}-, t\right), \mathbf{u}_{j}\left(a_{j}+, t\right)\right)$ with $i$ varying in $\operatorname{Inc}(J)$ and $j$ in Out $(J)$. 


\subsection{Waves Produced by Junctions}

This section describes the dynamics of the boundary data along roads meeting at a junction $J$. Thus, we fix a junction $J$ and, for simplicity, assume that $\operatorname{Inc}(J)=\{1, \ldots, n\}$ and $\operatorname{Out}(J)=\{n+1, \ldots, n+m\}$.

We begin with some notations.

Definition 6.5 $A$ datum $\mathbf{u}$ on an incoming road $I_{i}, i \in \operatorname{Inc}(J)$, is called bad if $\mathbf{u} \in \Omega_{f}$ and it is a called $\boldsymbol{g o o d}$ if $\mathbf{u} \in \Omega_{c}$.

A datum $\mathbf{u}$ on an outgoing road $I_{j}, j \in \operatorname{Out}(J)$, is called bad if $\mathbf{u} \in \Omega_{c}$ and it is a called good if $\mathbf{u} \in \Omega_{f}$.

The previous definition is justified as follows. We call good a datum if waves interacting with $J$ from other roads meeting at $J$ do not lead to phase change. Indeed, by the analysis of Section 4, we get:

Proposition 6.6 Let $\mathbf{u}_{0}=\left(\mathbf{u}_{1,0}, \ldots, \mathbf{u}_{n+m, 0}\right)$, i.e. R.S.J $\left(\mathbf{u}_{0}\right)=\mathbf{u}_{0}$ be an equilibrium at $J$ and assume that a wave is interacting with $J$.

If $\mathbf{u}_{k, 0}$ is good and the wave interacts from road $I_{k^{\prime}}$, with $k^{\prime} \neq k$, then the datum on $I_{k}$ after the interaction is still good and no phase boundary is produced on $I_{k}$. If $k \in \operatorname{Inc}(J)$ then only first family waves are produced, while if $k \in O u t(J)$ then only Lax waves in the free phase are produced.

If $\mathbf{u}_{i, 0}$ is bad, $i \in \operatorname{Inc}(J)$, and the wave interacts from road $I_{k}$, with $k \neq i$, then either no wave is produced on $I_{i}$ and the datum after the interaction is still $\mathbf{u}_{i, 0}$, or a phase boundary is produced on $I_{i}$ and the datum after the interaction is good.

If $\mathbf{u}_{j, 0}$ is bad, $j \in O u t(J)$, and the wave interacts from road $I_{k}$, with $k \neq j$, the datum after the interaction may be good or bad, with production on $I_{j}$ of possibly a second family wave, possibly a phase boundary and possibly a Lax wave in free phase.

A key point in obtaining the estimates on flux variation is to understand the type of waves which, after being produced by $J$, can come back to $J$ interacting only with waves produced by $J$.

Proposition 6.7 If a wave produced by $J$ on $I_{i}, i \in \operatorname{Inc}(J)$, come back to $J$ interacting only with waves produced by $J$ (and not with other junctions), then it is a phase boundary with decreasing flux connecting a left state in $\Omega_{f}$ to a right state in $\Omega_{c}$.

If a wave produced by $J$ on $I_{j}, j \in O$ Out $(J)$, comes back to $J$ interacting only with waves produced by $J$ (and not with other junctions), then either it is a phase boundary with decreasing flux connecting a left state in $\Omega_{f}$ to a right state in $\Omega_{c}$, or it is a Lax 1-wave in $\Omega_{c}$ with decreasing flux. 
Proof. Let us start considering $I_{i}, i \in \operatorname{Inc}(J)$. If a wave is produced by $J$ with a left good datum, then it is a first family wave in $\Omega_{c}$. The other waves produced by $J$ afterwards are necessarily first family waves as well by Proposition 6.6. Interaction with such waves produce again a first family wave, which has negative speed and thus can not come back to $J$. Then necessarily the left datum must be bad and the coming back wave is a phase boundary. The Rankine-Hugoniot condition with positivity of the velocity implies that the wave has decreasing flux.

Consider now the case of $I_{j}, j \in O u t(J)$. A phase boundary can come back to $J$ by interactions with other waves produced by $J$. However, in this case, the following can also happen. If a Lax wave in free phase is produced by $J$, then interacting with a phase boundary previously produced by $J$ can give rise to a first family wave in $\Omega_{c}$, which comes back to $J$. However, one can easily check that after the interaction with a phase boundary, the first family wave necessarily has decreasing flux. This property is kept if interactions with other first family waves with the same property or with other waves occur.

We now prove a lemma, used below to bound the number of waves.

Lemma 6.8 Assume that on the outgoing road $I_{j}$ there is no wave at time 0 , a phase boundary is produced on $I_{j}$ at time $t_{0}$ and comes back to $J$ at time $t_{1}$. Let $\mathbf{u}_{0, r}$ be the right state of the phase boundary at $t_{0}$. Assume that a Lax wave, emerging from $J$ in the free phase in the time interval $\left[t_{0}, t_{1}\right]$, interacts with the phase boundary and produces, a 1-wave in the congested phase coming back to $J$. Then, the total variation $T V_{f}^{J \rightarrow j}\left(\left[t_{0}, t_{1}\right]\right)$ of $\mathbf{f}_{1}$ computed on waves emerging from $J$ on road $I_{j}$ in the time interval $\left[t_{0}, t_{1}\right]$ satisfies

$$
T V_{f}^{J \rightarrow j}\left(\left[t_{0}, t_{1}\right]\right) \geq \frac{Q^{-}}{R}\left(R_{c}-\tilde{\rho}\right)>0 .
$$

Proof. Let $\mathbf{u}_{t}=\left(\rho_{t}, \rho_{t} V\right)$ be the intersection in the $(\rho, \rho v)$ plane of the tangent to $\Omega_{c}$ at $\mathbf{u}_{0, r}=\left(\rho_{0, r}, q_{0, r}\right)$ with $\Omega_{f}$. Then necessarily $T V_{f}^{J \rightarrow j}\left(\left[t_{0}, t_{1}\right]\right) \geq$ $\mathbf{f}_{1}\left(\mathbf{u}_{t}\right)-\mathbf{f}_{1}\left(\mathbf{u}_{0, r}\right)=\lambda_{1}\left(\mathbf{u}_{0, r}\right)\left(\rho_{t}-\rho_{0, r}\right)$ and the conclusion follows from the geometry of $\Omega_{f} \cup \Omega_{c}$ in the $(\rho, \rho v)$ plane (see Section 2.1 for the notation).

\subsection{Bounds on the Flux Variation}

This subsection is devoted to the estimate of the total variation of the density flux along an approximate wave-front tracking solution $\mathbf{u}_{\nu}$ and to the construction of solutions to the Cauchy problem.

First we fix $\mathbf{u}_{\nu}$, use notations (6.16)-(6.17), and assume that $\mathbf{u}_{\nu}$ has a finite number of waves at every time. We can then derive various total 
variation estimates. Then, using these total variation estimates, we prove that the number of waves is bounded and we can pass to the limit in $\nu$.

Lemma 6.9 Assume that a wave $\left(\mathbf{u}_{l}, \mathbf{u}_{r}\right)$ interacts with a junction $J$ at time $\bar{t}>0$. Then

$$
T V_{f}(\bar{t}+) \leq(C+1) T V_{f}(\bar{t}-) .
$$

where $C$ is given by property (Pr2).

Proof. By property (Pr2), we get

$$
T V_{f}(\bar{t}+)-T V_{f}(\bar{t}-) \leq C\left|\mathbf{f}_{1}\left(\mathbf{u}_{l}\right)-\mathbf{f}_{1}\left(\mathbf{u}_{r}\right)\right| .
$$

Therefore,

$$
T V_{f}(\bar{t}+) \leq T V_{f}(\bar{t}-)+C\left|\mathbf{f}_{1}\left(\mathbf{u}_{l}\right)-\mathbf{f}_{1}\left(\mathbf{u}_{r}\right)\right| \leq(C+1) T V_{f}(\bar{t}-)
$$

and this concludes the proof.

Lemma 6.10 Assume that a wave $\left(\mathbf{u}_{l}, \mathbf{u}_{r}\right)$ interacts with $J$ at a time $\bar{t}>0$. Then,

$$
\Gamma_{J}(\bar{t}+) \leq \Gamma_{J}(\bar{t}-)+(C+2)\left|\mathbf{f}_{1}\left(\mathbf{u}_{l}\right)-\mathbf{f}_{1}\left(\mathbf{u}_{r}\right)\right|,
$$

where $C$ is given by property (Pr2).

Proof. The variation of $\Gamma_{J}$ at $\bar{t}$ is the sum of the variation of the fluxes for the incoming roads. Therefore.

$$
\Gamma_{J}(\bar{t}+)-\Gamma_{J}(\bar{t}-) \leq T V_{f}(\bar{t}+)-T V_{f}(\bar{t}-)+2\left|\mathbf{f}_{1}\left(\mathbf{u}_{l}\right)-\mathbf{f}_{1}\left(\mathbf{u}_{r}\right)\right| .
$$

Hence, by (Pr2) and Lemma 6.9, the right hand side above is bounded by $(C+2)\left|\mathbf{f}_{1}\left(\mathbf{u}_{l}\right)-\mathbf{f}_{1}\left(\mathbf{u}_{r}\right)\right|$.

Lemma 6.11 There exists $C_{1}=C_{1}(\bar{v})$ such that, under assumption (H) the following holds. If a wave $\left(\mathbf{u}_{l}, \mathbf{u}_{r}\right)$ emerging from a junction $J$ reaches a different junction $J^{\prime}$ with states $\left(\mathbf{u}_{l}^{\prime}, \mathbf{u}_{r}^{\prime}\right)$, then

$$
\left|\mathbf{f}_{1}\left(\mathbf{u}_{l}^{\prime}\right)-\mathbf{f}_{1}\left(\mathbf{u}_{r}^{\prime}\right)\right| \leq C_{1}\left|\mathbf{f}_{1}\left(\mathbf{u}_{l}\right)-\mathbf{f}_{1}\left(\mathbf{u}_{r}\right)\right|
$$

Proof. It easily follows from the geometry of Lax waves in the congested phase $\Omega_{c}$.

Define:

$$
\delta=\left(\min _{i}\left|b_{i}-a_{i}\right|\right) /\left(\max \left\{V, \max _{\Omega_{c}}\left|\lambda_{1}\right|\right\}\right),
$$

which is the minimum time for a wave to travel from a junction to a different one. The next lemma gives a bound for the positive total variation of $\Gamma$. 
Lemma 6.12 If $t$ is not an interaction time, then we have

$$
\sum_{J \in \mathcal{J}} T V_{\Gamma_{J}}^{+}\left(\left[t, t+\delta[) \leq C_{1}(C+2) T V_{f}(t)\right.\right.
$$

where $C$ is given by property (Pr2), $C_{1}$ by Lemma 6.11 and $T V_{\Gamma_{J}}^{+}(\cdot)$ denotes the positive total variation (in time) of $\Gamma_{J}$.

Proof. By property (Pr3), an increment of the functional $\Gamma_{J}$ can happen only when a wave with increasing flux interacts with $J$. Moreover a wave, generated at $J$, can come back at $J$ only with a decreasing flux by Proposition 6.7.

By the definition of $\delta$, during the time interval $[t, t+\delta[$, a wave can not emerge from a junction $J$ and reach a different junction $J^{\prime}$. Thus we conclude, using lemmas 6.10 and 6.11 .

Lemma 6.13 For $C$ given by property (Pr2) and for any $t$ which is not an interaction time

$$
\sum_{J \in \mathcal{J}} T V_{\Gamma_{J}}\left(\left[t, t+\delta[) \leq 2 C_{1}(C+2) T V_{f}(t)+\#(\operatorname{Inc}(J)) F,\right.\right.
$$

where $T V_{\Gamma_{J}}$ indicates the total variation (in time) of $\Gamma_{J}$.

Proof. It is a direct consequence of Lemma 6.12 and of the bound (6.18).

With a slight abuse of notation, we set:

$$
\#(\mathcal{J})=\max _{J \in \mathcal{J}} \#(\operatorname{Inc}(J)) .
$$

Lemma 6.14 For every $t>0$ we have

$$
T V_{f}(t) \leq C_{2}^{\mathrm{CEIL}(t / \delta)} T V_{f}(0+)+C \#(\mathcal{J}) F \sum_{k=1}^{\mathrm{CEIL}(t / \delta)} C_{2}^{k},
$$

where $\operatorname{CEIL}(s)$ indicates the smallest integer greater than $s, C$ is given by property (Pr2), $C_{1}$ by Lemma 6.11 and $C_{2}=C_{1}(2 C(C+2)+1)$.

Proof. For simplicity, assume that $n \delta$ is not an interaction time for any positive integer $n$. Then, lemmas 6.13 and 6.11 and property (Pr2) imply:

$$
\begin{aligned}
T V_{f}((n+1) \delta) & \leq C \sum_{J \in \mathcal{J}} T V_{\Gamma_{J}}\left(\left[n \delta,(n+1) \delta[)+C_{1} T V_{f}(n \delta)\right.\right. \\
& \leq\left(2 C C_{1}(C+2)+C_{1}\right) T V_{f}(n \delta)+C \#(\mathcal{J}) F
\end{aligned}
$$


Setting $T V_{f, n}=T V_{f}(n \delta)$ and $C_{2}=\left(2 C C_{1}(C+2)+C_{1}\right)$, we can write:

$$
T V_{f, n+1} \leq C_{2} T V_{f, n}+C \#(\mathcal{J}) F .
$$

Then, by induction, we obtain the desired estimate.

Proof of Theorem 6.4. By Lemma 6.14 we have a bound on the density flux variation, as long as the approximate solution $\mathbf{u}_{\nu}$ is well defined.

By contradiction, assume that the number of waves blows up at a time $\bar{t}$. This may happen only due to interactions with a junction $J$. Fix $\xi>0$ such that in the interval $[\bar{t}-\xi, \bar{t}[$ all waves arriving at $J$ are produced by $J$ and coming back interacting only with waves produced by $J$.

We want first to prove that there is a finite number of possible values of $\mathbf{u}$ on each road in the time interval $[\bar{t}-\xi, \bar{t}[$ (possibly restricting $\xi$ ). Consider an incoming road $I_{i}$. All waves coming back to $J$ are phase boundaries, thus on $I_{i}$ either we are in the free phase or we are in the lower boundary of $\Omega_{c}$. In particular the maximal fluxes in the free phase and in the congested phase are constant. In a outgoing road $I_{j}$, the coming back waves can be either phase boundaries or Lax waves of the first family in $\Omega_{c}$. The latter may happen only if Lax waves in the free phase are produced by $J$, then interact with a phase boundary giving rise to the first family wave. In this case, by Lemma 6.8, the density flux variation produced by the outgoing Lax wave on $I_{j}$ (from $J$ ) is bounded away from zero. As a consequence, by Lemma 5.1, a bounded away from zero variation (in time) of $\Gamma_{J}$ occurs. Thus this can not happen an infinite number of times because of Lemma 6.13. Finally, possibly restricting $\xi$, we can assume that only phase boundaries are coming back to $J$ and thus also on outgoing roads the maximal fluxes in the free phase and in the congested phase are constant (the right state in the congested phase being fixed). By property (Pr1) there are only a finite number of possible solutions emerging from $J$.

Since there are only a finite number of possible values at $J$, any wave interaction with $J$ that produces waves on other roads would produce a bounded away from zero change in $\Gamma_{J}$ (again by Lemma 5.1). Therefore, infinite oscillations among these values are not possible, due again to Lemma 6.13. Interactions that do not produce flux variations on other roads do not increase the number of waves.

We proved that $\mathbf{u}_{\nu}$ is well defined for every time. The number of phase boundaries in $\mathbf{u}_{\nu}(0)$ is bounded by $T V\left(\mathbf{u}_{\nu}(0)\right) / \varepsilon$, where $\varepsilon$ is the minimal variation of a phase boundary. Then new phase boundaries can be produced by each junction $J$, but with at most one phase boundary on each road for each junction, due to Proposition 6.6. Now, by Lemma 6.14 and Proposition 6.1 we get $\mathbf{B V}$ estimates on $\rho_{\nu}$ and $q_{\nu}$. Indeed, since the maximal $\rho$ 
variation of a phase boundary is bounded by $R$, we can write:

$$
T V\left(\rho_{\nu}(t)\right) \leq C T V_{f}(t)+R\left(\frac{T V\left(\mathbf{u}_{\nu}(0)\right)}{\varepsilon}+2 N\right),
$$

where $C=C(\bar{v})$ is as in Proposition 6.1 and $N$ is the number of roads of the network; similarly for $T V\left(q_{\nu}\right)$. Passing to the limit in $\nu$, the proof is concluded.

\section{Appendix}

In this Appendix we give an example showing that violating assumption $(\mathbf{H})$ may cause an unbounded increase in the variations of $\rho$ and $q$.

Consider a simple junction with one incoming road $\left.\left.I_{1}=\right]-\infty, 0\right]$ and one outgoing road $I_{2}=\left[0,+\infty\left[\right.\right.$. We assume that on $I_{1}$ the initial condition $\mathbf{u}_{1,0}$ is always in the free phase and satisfies

$$
\rho_{1,0}(x)=\vartheta+\sum_{i=1}^{+\infty} \varepsilon_{i} \chi_{\left[x_{2 i+1}, x_{2 i}[\right.},
$$

where $\vartheta>0, \varepsilon_{i}>0$ are sufficiently small with $\sum_{i} \varepsilon_{i}=1, \chi$ is the indicator function and $x_{i}$ is a negative decreasing sequence. On $I_{2}$ the constant initial condition $\mathbf{u}_{2,0}$ is on the lower boundary of $\Omega_{c}$ and satisfies:

$$
\mathbf{u}_{2,0} v_{c}\left(\mathbf{u}_{2,0}\right)=\vartheta v_{f}(\vartheta) .
$$

In particular, at time 0 there is an equilibrium at the junction and the initial datum $\mathbf{u}_{1,0}$ has bounded total variation. For a suitable choice of the sequence $x_{i}$, all waves in $I_{1}$ reach the junction before interacting among each other (it is easy to verify that the sequence $x_{i}$ is necessarily unbounded). For $\varepsilon_{i}$ sufficiently small, the state in $I_{2}$ remains on the curve of the second family through $\mathbf{u}_{2,0}$ and the waves produced on $I_{2}$ all have the same velocity $v_{c}\left(\mathbf{u}_{2,0}\right)$, thus they do not interact on $I_{2}$. After the $n$-th interaction of waves from $I_{1}$ with the junction, the $\rho$ variation on $I_{2}$ is

$$
\frac{1}{v_{c}\left(\mathbf{u}_{2,0}\right)} \sum_{i=1}^{n} V \varepsilon_{i}\left(1-\frac{\varepsilon_{i}+2 \vartheta}{R}\right) \text {. }
$$

Now, as $\vartheta \rightarrow 0$, the term $1 / v_{c}\left(\mathbf{u}_{2,0}\right)$ tends to infinity, while the sum can be kept bounded away from zero by a suitable choice of $n=n(\vartheta)$. The total variation of $\rho$ can become arbitrarily large and the same happens for the total variation of $q$.

Acknowledgments. The second author was partially supported by I.N.R.I.A. Sophia Antipolis - Méditerranée. 


\section{References}

[1] A. Aw and M. Rascle. Resurrection of "second order" models of traffic flow. SIAM J. Appl. Math., 60:916-938, 2000.

[2] M. K. Banda, M. Herty, and A. Klar. Gas flow in pipeline networks. Netw. Heterog. Media, 1(1):41-56 (electronic), 2006.

[3] G. Bastin and B. Haut. A second order model of road junctions in fluid models of traffic networks. Netw. Heterog. Media, 2(2):227-253 (electronic), 2007.

[4] A. M. Bayen, D. Sun, and I. S. Strub. Comparison of the performance of four Eulerian network flow models for strategic air traffic management. Netw. Heterog. Media, 2(4):569-595 (electronic), 2007.

[5] N. Bellomo and V. Coscia. First order models and closure of the mass conservation equation in the mathematical theory of vehicular traffic flow. C.R. Mecanique, 333:843-851, 2005.

[6] A. Bressan. Hyperbolic systems of conservation laws, volume 20 of Oxford Lecture Series in Mathematics and its Applications. Oxford University Press, Oxford, 2000. The one-dimensional Cauchy problem.

[7] Y. Chitour and B. Piccoli. Traffic circles and timing of traffic lights for cars flow. Discrete Contin. Dyn. Syst. Ser. B, 5(3):599-630, 2005.

[8] G. M. Coclite, M. Garavello, and B. Piccoli. Traffic flow on a road network. SIAM J. Math. Anal., 36(6):1862-1886 (electronic), 2005.

[9] R. M. Colombo. Hyperbolic phase transitions in traffic flow. SIAM J. Appl. Math., 63(2):708-721, 2002.

[10] R. M. Colombo. Wave front tracking in systems of conservation laws. Appl. Math., 49(6):501-537, 2004.

[11] R. M. Colombo and M. Garavello. A well posed Riemann problem for the p-system at a junction. Netw. Heterog. Media, 1(3):495-511 (electronic), 2006.

[12] R. M. Colombo and M. Garavello. On the Cauchy problem for the $p$-system at a junction. SIAM J. Math. Anal., 39(5):1456-1471, 2008.

[13] R. M. Colombo, P. Goatin, and F. S. Priuli. Global well posedness of a traffic flow model with phase transitions. Nonlinear Anal. Ser. A, 66:2413-2426, 2007.

[14] C. M. Dafermos. Hyperbolic conservation laws in continuum physics, volume 325 of Grundlehren der Mathematischen Wissenschaften [Fundamental Principles of Mathematical Sciences]. Springer-Verlag, Berlin, 2000.

[15] C. D'Apice and R. Manzo. A fluid dynamic model for supply chains. Netw. Heterog. Media, 1(3):379-398 (electronic), 2006.

[16] C. D'Apice, R. Manzo, and B. Piccoli. Packet flow on telecommunication networks. SIAM J. Math. Anal., 38(3):717-740 (electronic), 2006.

[17] M. Garavello, R. Natalini, B. Piccoli, and A. Terracina. Conservation laws with discontinuous flux. Netw. Heterog. Media, 2(1):159-179 (electronic), 2007. 
[18] M. Garavello and B. Piccoli. Source-destination flow on a road network. Commun. Math. Sci., 3(3):261-283, 2005.

[19] M. Garavello and B. Piccoli. Traffic flow on a road network using the AwRascle model. Comm. Partial Differential Equations, 31(1-3):243-275, 2006.

[20] M. Garavello and B. Piccoli. Traffic flow on networks, volume 1 of AIMS Series on Applied Mathematics. American Institute of Mathematical Sciences (AIMS), Springfield, MO, 2006. Conservation laws models.

[21] M. Garavello and B. Piccoli. Conservation laws on complex networks. Annales de l'Institut Henri Poincaré - Analyse Non Linéaire, To appear.

[22] M. Garavello and B. Piccoli. On fluido-dynamic models for urban traffic. Netw. Heterog. Media, To appear.

[23] P. Goatin. The Aw-Rascle traffic flow model with phase transition. Math. Comput. Modeling, 44:287-303, 2006.

[24] S. Göttlich, M. Herty, and A. Klar. Network models for supply chains. Commun. Math. Sci., 3(4):545-559, 2005.

[25] J. M. Greenberg, A. Klar, and M. Rascle. Congestion on multilane highways. SIAM J. Appl. Math., 63(3):818-833 (electronic), 2003.

[26] D. Helbing. Improved fluid-dynamic model for vehicular traffic. Phys. Rev. E, 51(4):3164-3169, 1995.

[27] D. Helbing. Traffic and related self-driven many-particle systems. Rev. Mod. Phys., 73:1067-1141, 2001.

[28] D. Helbing, J. Siegmeier, and S. Lämmer. Self-organized network flows. Netw. Heterog. Media, 2(2):193-210 (electronic), 2007.

[29] M. Herty, S. Moutari, and M. Rascle. Optimization criteria for modelling intersections of vehicular traffic flow. Netw. Heterog. Media, 1(2):275-294 (electronic), 2006.

[30] H. Holden and N. H. Risebro. A mathematical model of traffic flow on a network of unidirectional roads. SIAM J. Math. Anal., 26(4):999-1017, 1995.

[31] H. Holden and N. H. Risebro. Front tracking for hyperbolic conservation laws, volume 152 of Applied Mathematical Sciences. Springer-Verlag, New York, 2002.

[32] M. J. Lighthill and G. B. Whitham. On kinematic waves. II. A theory of traffic flow on long crowded roads. Proc. Roy. Soc. London. Ser. A., 229:317-345, 1955.

[33] H. J. Payne. Models of freeway traffic and control. In Simulation Council Proc.28, pages 51-61. Math. Models Publ. Sys., 1971.

[34] P. I. Richards. Shock waves on the highway. Operations Res., 4:42-51, 1956.

[35] F. Siebel and W. Mauser. On the fundamental diagram of traffic flow. SIAM J. Appl. Math., 66(4):1150-1162 (electronic), 2006.

[36] B. Temple. Systems of conservation laws with coinciding shock and rarefaction curves. Contemp. Math., 17:143-151, 1983. 
[37] G. B. Whitham. Linear and nonlinear waves. Wiley-Interscience [John Wiley \& Sons], New York, 1974. Pure and Applied Mathematics.

[38] H. M. Zhang. A non-equilibrium traffic model devoid of gas-like behavior. Transport. Res. B, 36(5):275-290, 2002. 